\title{
Erratum on "Boundary Conditions for Scalar Conservation Laws, from a Kinetic Point of View"
}

\section{A. Nouri, A. Omrane, and J. P. Vila}

This note corrects a Gronwall argument in the proof of the uniform $L^{\infty}$ bound on $u_{\epsilon}(t, x):=\int f_{\epsilon}(t, x, v) d v$ given in ref. 2 . The main result of the paper, i.e., Theorem 1 , still holds and now even in an appropriate $L^{\infty}$ setting not requiring the earlier $B V$ assumption of ref. 2 . In the statement of Theorem 1, "Under some technical assumptions (of Proposition 4), the function $u_{\epsilon}$ converges in $L^{\infty}\left(0, T ; L_{\text {loc }}^{1}(\Omega)\right)$ to a function $u \in L^{\infty}(0, T$; $B V(\Omega)) \ldots$.." should be replaced by "Under the assumption $\mid\{v \in \mathbb{R} ; a(v) \cdot \sigma$ $=u\} \mid=0, \sigma \in S^{N-1}, u \in \mathbb{R}$ and in the setting of Theorem 2, the function $u_{\epsilon}$ converges in $L^{\infty}\left(0, T ; L_{\mathrm{loc}}^{1}(\Omega)\right)$ to a function $u \in L^{\infty}((0, T) \times \Omega) \ldots$. ." Theorem 2 should be completed by the following.

Finally, under the assumption that $f_{0}$ and $\tilde{f}$ are $L^{\infty}$ functions with $\left\|f_{0}\right\|_{L^{\infty}} \leqslant 1,\|\tilde{f}\|_{L^{\infty}} \leqslant 1, f_{0}(\cdot, v) \operatorname{sgn}(v) \geqslant 0, \tilde{f}(\cdot, v) \operatorname{sgn}(v) \geqslant 0$, and compact supports in $v, u_{\epsilon}$ is bounded in $L^{\infty}((0, T) \times \Omega)$ uniformly w.r.t. $\epsilon$.

The following is a proof for $\Omega=] 0,+\infty$ [. In the proof of Theorem 2, a Banach fixed point argument in $L^{\infty}\left(0, T ; L^{1}(\Omega \times \mathbb{R})\right)$ was performed for the map $\mathscr{T}$ that maps $f$ into $F$ solution to $\partial_{t} F+a_{1}(v) \partial_{x} F=\frac{1}{\epsilon}\left(\chi_{u_{f}}-F\right)$ with the same initial and boundary data as in (10). Under the previous assumptions on $f_{0}$ and $\tilde{f}$, there is a positive number $M$ such that $\chi_{-M}(v) \leqslant$ $f_{0}(x, v) \leqslant \chi_{M}(v)$ and $\chi_{-M}(v) \leqslant \tilde{f}(t, v) \leqslant \chi_{M}(v)$. Prove that if $\left\|u_{f}\right\|_{\infty} \leqslant M$ then $\left\|u_{\mathscr{T}(f)}\right\|_{\infty} \leqslant M$.

For $x-t a_{1}(v)>0$,

$$
F(t, x, v)=f_{0}\left(x-t a_{1}(v), v\right) e^{-\frac{t}{\epsilon}}+\int_{0}^{t} \frac{1}{\epsilon} e^{\frac{s-t}{\epsilon}} \chi_{u_{f}\left(s, x+(s-t) a_{1}(v)\right)}(v) d s .
$$

For $x-t a_{1}(v)<0$,

$$
F(t, x, v)=\tilde{f}\left(t-\frac{x}{a_{1}(v)}, v\right) e^{-\frac{x}{\epsilon a_{1}(v)}}+\int_{t-\frac{x}{a_{1}(v)}}^{t} \frac{1}{\epsilon} e^{\frac{s-t}{\epsilon}} \chi_{u_{f}\left(s, x+(s-t) a_{1}(v)\right)}(v) d s .
$$


And so,

$$
\begin{aligned}
\int F(t, x, v) d v \leqslant & e^{-\frac{t}{\epsilon}} \int_{a_{1}(v)<\frac{x}{t}} f_{0}\left(x-t a_{1}(v), v\right) d v \\
& +\int_{0}^{t} \frac{1}{\epsilon} e^{\frac{s-t}{\epsilon}} \int_{a_{1}(v)<\frac{x}{t}} \chi_{u_{f}\left(s, x+(s-t) a_{1}(v)\right)}(v) d v d s \\
& +\int_{a_{1}(v)>\frac{x}{t}} \tilde{f}\left(t-\frac{x}{a_{1}(v)}, v\right) e^{-\frac{x}{\epsilon a_{1}(v)}} d v \\
& +\int_{a_{1}(v)>\frac{x}{t}} \int_{t-\frac{x}{a_{1}(v)}} \frac{1}{\epsilon} e^{\frac{s-t}{\epsilon}} \chi_{u_{f}\left(s, x+(s-t) a_{1}(v)\right)}(v) d s d v \\
\leqslant & e^{-\frac{t}{\epsilon}} \int_{a_{1}(v)<\frac{x}{t}} \chi_{M}(v) d v+\left(1-e^{-\frac{t}{\epsilon}}\right) \int_{a_{1}(v)<\frac{x}{t}} \chi_{M}(v) d v \\
& +\int_{a_{1}(v)>\frac{x}{t}} \chi_{M}(v) e^{-\frac{x}{\epsilon a_{1}(v)}} d v \\
& +\int_{a_{1}(v)>\frac{x}{t}} \chi_{M}(v)\left(1-e^{-\frac{x}{\epsilon a_{1}(v)}}\right) d v=M .
\end{aligned}
$$

Similarly, $\int F(t, x, v) d v \geqslant-M$.

Proposition 3(iii), Propositions 4 and 5 are skipped. The second and third lines of p. 795 no more follow from Proposition 5 but from the strong convergence in $L^{1}$ of $f_{\epsilon}$ to $\chi_{u}$ derived from now classical arguments. Indeed, $\left(f_{\epsilon}\right)$ being uniformly bounded in $L^{1} \cap L^{\infty}$, converges (up to a subsequence) to some $f$ in $L^{2}$ weak. By an averaging lemma (ref. 1, p. 23), $u_{\epsilon}$ converges strongly in $L^{1}$ to $u(t, x):=\int f(t, x, v) d v$. By the integral representation of $f_{\epsilon}, f_{\epsilon}$ converges strongly in $L^{1}$ to $\chi_{u}$ (ref. 3, p. 81). The reference to Helly's theorem p. 795 should be skipped. The rest of the paper is unchanged.

\section{REFERENCES}

1. F. Bouchut, F. Golse, and M. Pulvirenti, Kinetic Equations and Asymptotic Theory (Gauthier-Villars, 2000).

2. A. Nouri, A. Omrane, and J. P. Vila, Boundary conditions for scalar conservation laws from a kinetic point of view, J. Stat. Phys. 94:779-804 (1999).

3. B. Perthame, Kinetic Formulations of Conservation Laws (Oxford University Press, Oxford, 2002). 